\title{
WPVS 007 and Mkn 335: the link between NLS1s and BAL QSOs
}

\section{Dirk Grupe*}

Pennsylvania State University, 525 Davey Lab, University Park, PA 16802, USA

E-mail: grupedastro.psu.edu

\section{S. Komossa}

Technische Universitaet Muenchen, Lehrstuhl fuer Physik I, James Franck Strasse 1/I, 85748

Garching, Germany; Excellence Cluster Universe, TUM, Boltzmannstrasse 2, 85748 Garching,

Germany; Max Planck Institut fuer Plasmaphysik, Boltzmannstrasse 2, 85748 Garching,

Germany

E-mail: stefanie.komossaegmx.de

\section{Karen Leighly}

University Of Oklahoma, 440 West Brooks Street, Norman, OK 73019, USA

E-mail: leighlyenhn.ou.edu

\section{Luigi Gallo}

Saint Mary's University, Halifax, NS B3H 3C3, Canada

E-mail: lgallodap.stmarys.ca

The X-ray transient AGN WPVS 007 was an X-ray bright source during the ROSAT All-Sky Survey, but then basically disappeared from the X-ray sky for almost two decades. FUSE observations in 2003 revealed the nature of this disappearance: strong absorption. FUSE spectra showed deep absorption line troughs in the UV spectrum of WPVS 007. These findings make this AGN a direct link between NLS1s and Broad Absorption Line QSOs which are both thought to be high $\mathrm{L} / \mathrm{L}_{\text {edd }}$ AGN. In recent years Swift was able to detect WPVS 007 in X-rays at relatively bright states several times, suggesting that the absorber may have started to disappear or become more leaky. In some ways similar is the NLS1 Mkn 335 which has been observed in X-rays in a bright state over decades, but was found by Swift in an extremely low state in May 2007. Our XMM observation of Mkn 335 from June 2009 is well fit by the presence of a strong ionized partial covering absorber.

Narrow-Line Seyfert 1 Galaxies and their place in the Universe - NLS1, April 04-06, 2011

Milan Italy

\footnotetext{
* Speaker.
} 


\section{Introduction}

Narrow Line Seyfert 1 galaxies are the most variable non-beamed AGN in X-rays. Typically, AGN vary by factors of about three even within days. However, the NLS1 WPVS 007 [औ is a unique X-ray transient AGN: While typically X-ray transience is associated with an X-ray outburst caused by a dramatic increase in the accretion rate, the situation in WPVS 007 is different. Here the AGN was bright during the ROSAT All-Sky Survey, but then basically disappeared from the $\mathrm{X}$-ray sky ever since. The cause of this transience had been a mystery for more than a decade, but observations with FUSE revealed that it is a strong absorber that causes the AGN to be so X-ray faint [10].

Somewhat similar is the NLS1 Mkn 335. This AGN has been known to be X-ray bright since it was first detected by UHURU. However, when we observed it in May 2007 with Swift, we discovered it in an extremely low X-ray flux state. A follow-up observation with XMM showed that one possible explanation for this low state is a strong partial covering absorber.

\section{Observations of WPVS 007 and Mkn 335}

We have been monitoring WPVS 007 with Swift since October 2005 [5], 8] at a frequency of typically once per month for $2 \mathrm{ks}$ with the XRT and and UVOT in all 6 filters. This is still an ongoing monitoring campaign of which the exposure time has been increased to $5 \mathrm{ks}$ per observation since April 2010.

Mkn 335 was observed as part of a Swift fill-in program to study the spectral energy distributions of AGN [9]. After it was discovered to be in an extreme low X-ray flux state we have monitored it for at least once per month except for the three months when it cannot be observed by Swift becasue of the Sun-constraint.

\section{X-ray Variability of WPVS 007}

Figure 1 displays the long-term 0.2-2.0 keV flux light curve of WPVS 007. This NLS1 has shown the most dramatic decrease of any known AGN in X-rays by a factor of about 400 between the ROSAT All-Sky Survey (RASS) observation in 1990 and a pointed ROSAT observation in 1993 [円]. Follow up observations with ROSAT and Chandra confirmed this extreme low state. We were finally able to detect it again in X-rays with Swift in a $50 \mathrm{ks}$ observation in September 2007. This observation also revealed for the first time hard X-ray photons from this AGN that had the steepest X-ray spectrum of any AGN during the RASS. The photon distribution during this observation could be fitted by a power law model with a strong partial covering absorber [8].

\section{WPVS 007: The link between NLS1s and Broad Absorption Line QSOs}

The dramatic decrease in X-rays by a factor of more than 400 between 1990 and 1993 was puzzling. HST UV spectra taken in 1996 [3] showed mini Broad Absorption Lines (BALs) suggesting outflowing absorbing matter. However, when FUSE observed WPVS 007 in the FUV in 2003 it became clear what is going on in this AGN: This FUV spectrum as shown in Figure 2 


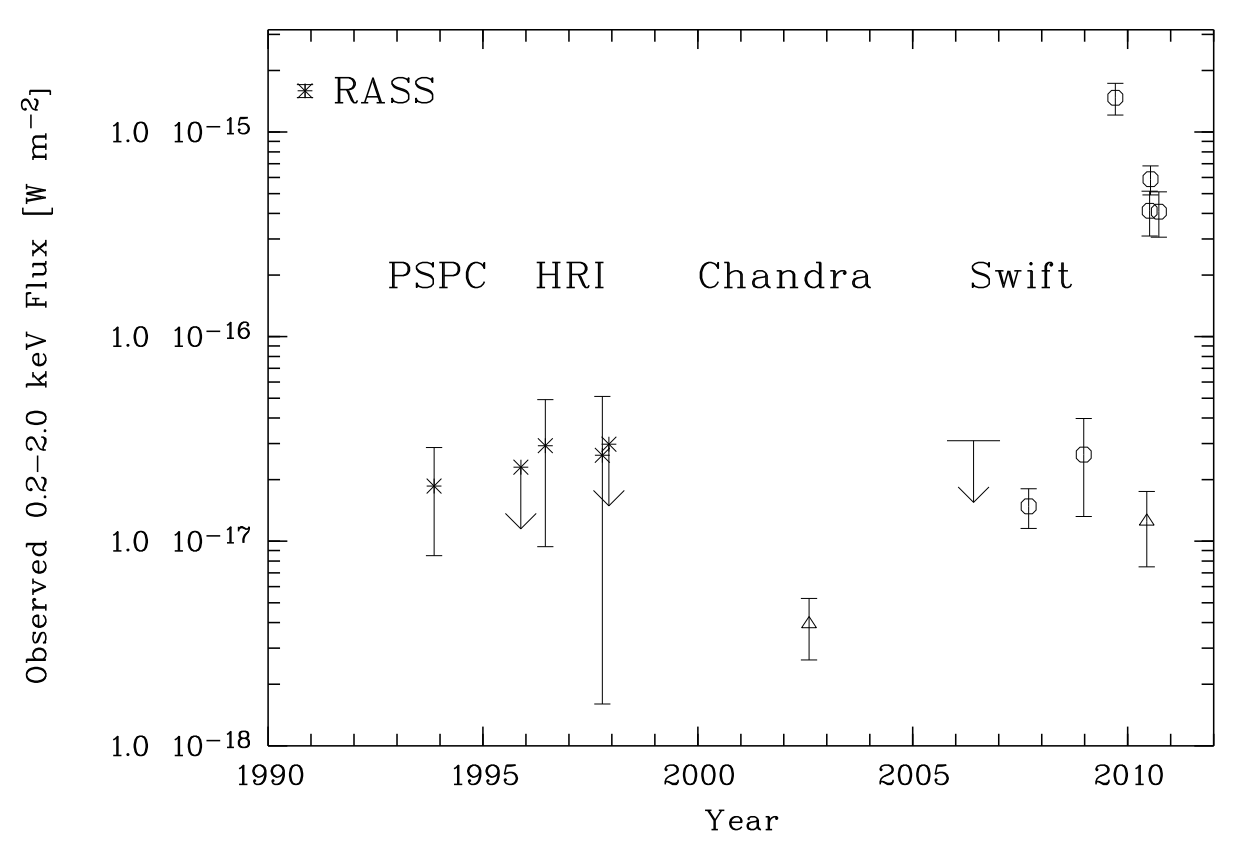

Figure 1: WPVS 007 long-term 0.2-2.0 keV X-ray flux light curve.

revealed very strong BALs, representing the discovery of the emergence of BALs for the first time in a low-luminosity AGN [10]. It has been suggested by e.g. [2] and [1] that NLS1s and BAL QSOs are closely related, being both AGN that operate at high L/L edd ratios. WPVS 007 is the link between these two AGN classes.

Figure 3 displays the Swift UVOT light curves of WPVS 007 from October 2005 to March 2011. WPVS 007 is highly variable in the UV with up to 0.7 mag in W2. Typically NLS1s only show UV variability on the order of 0.3 mag [9]. We will continue to monitor WPVS 007 in the future with Swift in order to catch it when it will be in an X-ray high flux state again, and in order to study how this flux state relates to the UV emission.

\section{Swift and XMM Observations of Mkn 335}

Although the NLS1 Mkn 335 has been known to be an X-ray bright AGN since its first detection in X-rays by UHURU, when we observed it with Swift as part of our fill-in program to study the SEDs of AGN [9] we discovered it in an extreme X-ray low flux state [6]. We have monitored 


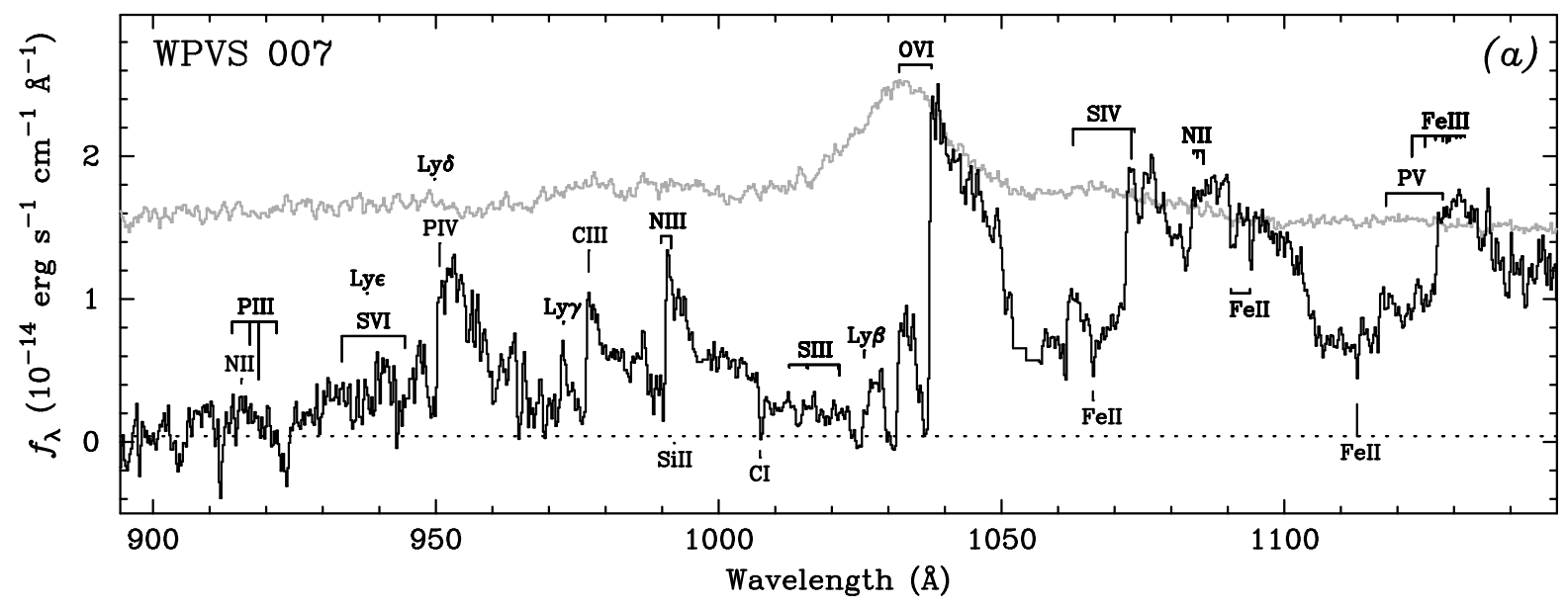

Figure 2: FUSE spectrum of WPVS 007 [10]. in comparison with the HST composite spectrum (grey line; [12] ]). Galactic absorption lines are marked below the spectrum, and the rest wavelengths of possible absorption lines are marked above the spectrum.

Mkn 335 with Swift on a monthly basis and found it to be highly variable in X-rays as well as in the UV (Figure 4). However, although it has shown an episode of being in a relatively high state, for most of the time it has remained in a low X-ray flux state. Our XMM observation from July 2007 (Figure 5) showed that the X-ray spectrum can be fitted by a partial covering absorber [7]. We had another XMM observation in June 2009 which suggests that the partial covering absorber has become ionized. Alternatively the X-ray spectra can also be fitted by reflection models (Grupe et al. 2011, in prep.).

The dramatic drop in X-ray flux by a factor of more than 30 between 2006 and 2007 [6] and 


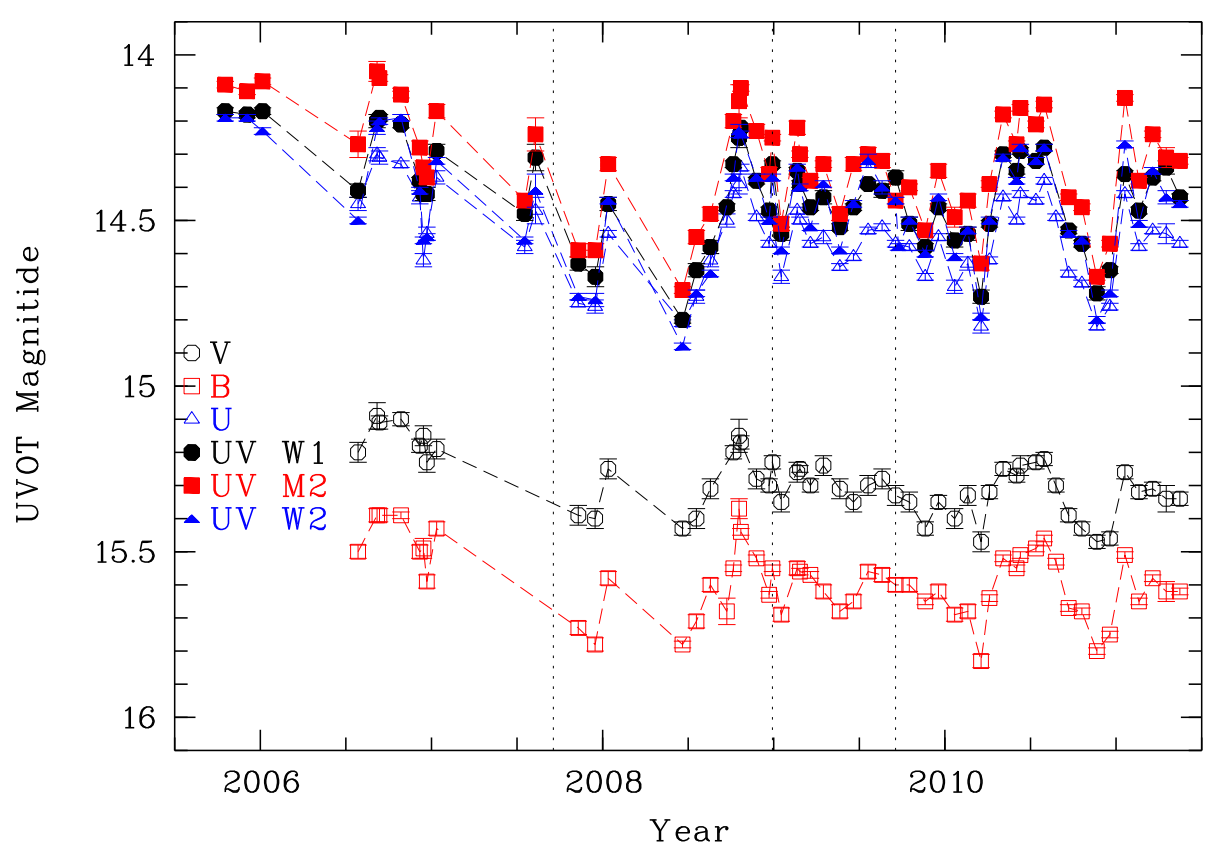

Figure 3: Swift UVOT light curves of WPVS 007 starting October 2005.

the possible explanation of the X-ray spectrum with a partial covering absorber may suggest that we see a similar process in Mkn 335 that we see in WPVS 007.

\section{References}

[1] Boroson, T.A., 2002, ApJ, 565, 78

[2] Brandt, W.N., \& Gallagher, S.C., 2000, New Astronomy Review, 44, 461

[3] Goodrich, R.W., 2000, New Astronomy Review, 44, 519

[4] Grupe, D., et al., 1995, A\&A, 300, L21

[5] Grupe, D., et al., 2007a, AJ, 133, 1988

[6] Grupe, D., Komossa, S., \& Gallo, L.C., 2007b, ApJ 681, 982

[7] Grupe, D., et al., 2008a, ApJ, 681, 982

[8] Grupe, D., Leighly, K.M., \& Komossa, S., 2008b, AJ, 136, 2343 


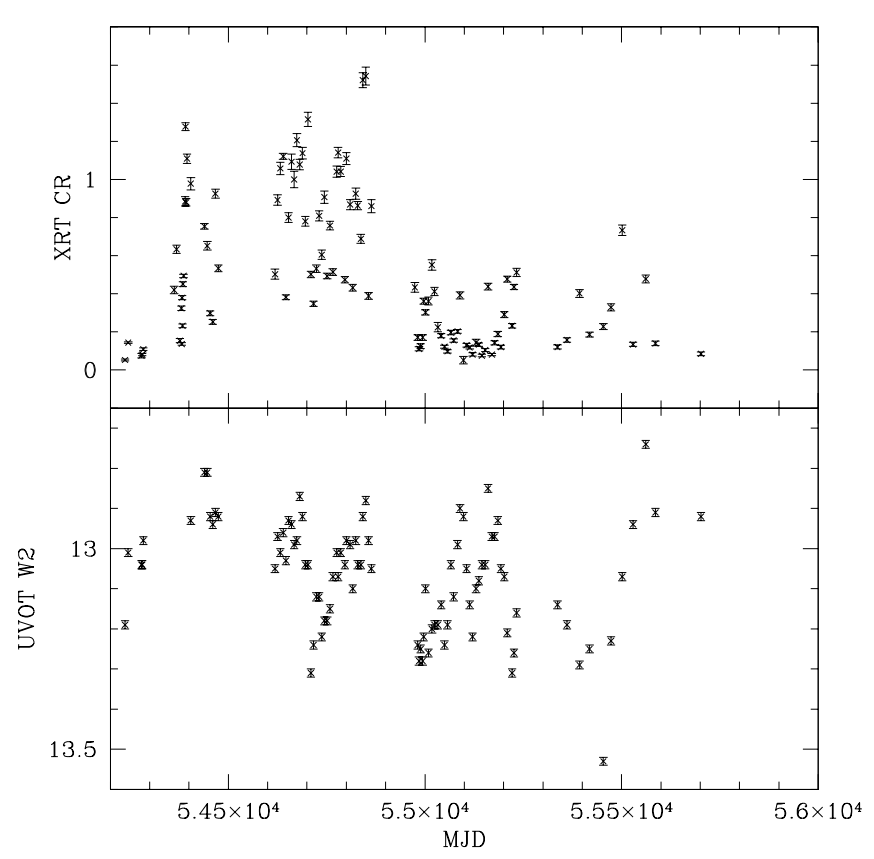

Figure 4: Swift XRT count rate and UVOT W2 light curves of Mkn 335 (Grupe et al. 2011, in prep.).

[9] Grupe, D., Komossa, K.M., Leighly, K.M., \& Page, K.L., 2010, ApJS, 187, 64

[10] Leighly, K.M., Hamann, F., Casebeer, D.A., \& Grupe, D., 2009, ApJ, 701, 176

[11] Tueller, J., et al., 2010, ApJS, 186, 378

[12] Zheng, W., et al., 1997, ApJ, 475, 469

\section{Acknowledgments}

Swift is supported at Penn State by NASA contract NAS5-00136. This research has been supported by NASA contracts NNX09AP50G, NNX08AT25G, NNX10AAF48G and NNX10AK85G. 


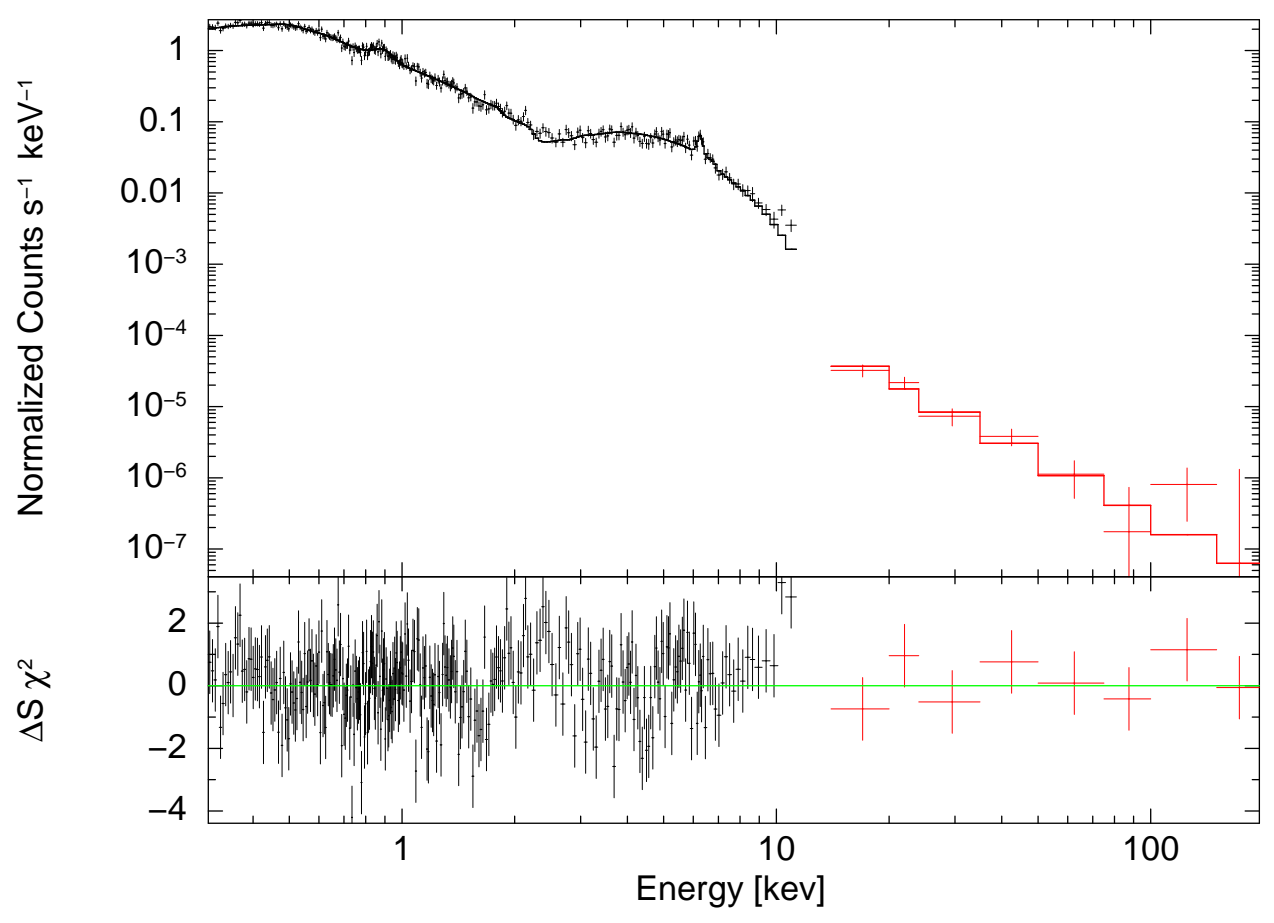

Figure 5: Combined XMM Epic pn and Swift BAT spectra of Mkn 335 [7] from the 2007 XMM observation and the 22 months BAT survey [11]. 